\title{
Predictive Values for Molecular Diagnostics: Converting Unknown Unknowns to Known Unknowns
}

\author{
Cathal P. O'Brien - Stephen E. Langabeer • \\ Kenneth J. O'Byrne · John J. O'Leary • \\ Stephen P. Finn
}

Published online: 12 December 2013

(c) Springer International Publishing Switzerland 2013

\section{Introduction}

While molecular diagnostics are a relatively recent development in the field of oncology, the utility of this limb of pathology is undeniable. Prototypic companion diagnostics treatments are widely described in the fields of oncology and pathology; for example, KRAS mutation testing prior to anti-epidermal growth factor receptor (EGFR) monoclonal antibody therapy (cetuximab, panitumumab) for metastatic colorectal cancer (mCRC), or EGFR mutation testing in stage IIIB and stage IV non-small-cell lung cancer (NSCLC) to establish suitability for gefitinib and erlotinib therapy.

In the case of predictive mutation testing for oncology, the results of a single set of examinations (molecular markers) decide a patient's eligibility for therapy. This places on the treating oncologist an absolute dependence on a single set of investigations to decide a patient's

C. P. O’Brien $(\varangle) \cdot$ S. E. Langabeer · S. P. Finn

Cancer Molecular Diagnostics, Central Pathology Laboratory,

St. James's Hospital, Dublin 8, Ireland

e-mail: obriec12@tcd.ie

C. P. O'Brien · J. J. O'Leary · S. P. Finn

Department of Histopathology, St James's Hospital, Dublin 8, Ireland

C. P. O’Brien · J. J. O'Leary · S. P. Finn

Department of Histopathology, Trinity College Dublin,

Dublin 8, Ireland

K. J. O’Byrne

Department of Oncology, St James's Hospital, Dublin 8, Ireland

J. J. O'Leary

Department of Histopathology, The Coombe Women

and Children's Hospital, Dublin 8, Ireland treatment options. However, as some authors have already demonstrated, our intuitive grasp of probabilities can lead to a false level of confidence in the veracity of medical results [1], and predictive mutation assays are no exception to this rule. As Gigerenzer and colleagues have demonstrated, this false confidence can stem from a lack of information on, or understanding of, the positive or negative predictive value of an assay. This problem may be compounded where the same mutation may be found in multiple disorders and may predict favourable response to therapy for one cancer type and may contra-indicate it for another.

\section{The Mathematical Basis of Predictive Values}

To illustrate the difference between sensitivity and specificity and the predictive value of an investigation, we will first discuss a theoretical mutation detection assay with a sensitivity of 0.90 and a specificity of 0.95 , where a positive mutation result is required to institute a therapeutic option.

While the above sensitivity and specificity values may appear acceptable, calculation of the predictive values of the assay tell a different story. If one imagines that the mutation being detected by the theoretical assay described above is quite rare in the population being tested, 1 in 100 for example, the positive predictive value (PPV) can be calculated as follows: with a prevalence in our tested population of 1 in 100 , for every 1,000 patients tested, 10 will have the mutation of interest and 990 will not. With a sensitivity of $90 \%$ it is likely that 9 of the 10 true positives will be detected. However, the remaining 990 mutationnegative patients will also be tested, what of their results? Quite simply, our theoretical assay with a specificity of 
0.95 will have a false positive rate of $0.05(5 \%)$.This will result in approximately 50 false positives $(990 \times$ $0.05)$. Thus, for every 1,000 patients screened for this mutation with this assay, we will obtain 59 positive results. True positives will account for 9 of the positive results and the remaining 50 positive results will be false positives (Fig. 1). This can be calculated to give a PPV of $15.25 \%$. Or, to put it another way, a positive result is likely to be indicative of a true patient positive in $15.25 \%$ of cases. Calculation of the negative predictive value (NPV) would yield a more acceptable $99.9 \%$ (see Fig. 1). From the figures above, it can be clearly seen that the prevalence of the disease can directly affect the probability that the patient's result does correspond to a true positive. From the intuitive presumption of a positive result being $90 \%$ likely to indicate a true positive result, we are reduced to the comparably poor likelihood of $15.25 \%$.

\section{Relevance of Predictive Values in a Diagnostic Setting}

While the previous example is one of a theoretical assay and disease, clinical scenarios already exist where these principles are of direct relevance, as is the case with $B R A F$ mutation testing. Testing for mutations of the $B R A F$ gene is a pre-requisite for prescription of vemurafinib for the treatment of malignant melanoma. This serine-threonine kinase inhibitor has been shown to extend the lifespan of stage III and stage IV malignant melanoma patients, and has recently been approved by the European Medicines Agency (EMA) for prescription in un-resectable or metastatic melanoma where the tumour exhibits a mutation at codon 600 of the BRAF gene [2]. Thus, once a patient's tumour has been shown to contain an activating mutation of the BRAF gene, specifically a p.V600X-generating mutation, it is likely that treatment with vemurafinib will
Fig. 1 Illustration of the combined effect of disease prevalence and assay sensitivity and specificity on the positive and negative predictive value of an assay. The figure illustrates a starting population of 1,000 patients; 10 patients harbour the mutation being examined by the assay, the remaining 990 patients do not. Based on an assay specificity of $95 \%, 940$ patients will be correctly classified as not carrying the mutation $(A), 50$ patients will be incorrectly classified as carrying the mutation $(C)$. The assay sensitivity of $90 \%$ means that 1 of the 10 mutation-carrying patients will be incorrectly classified as not carrying the mutation $(B)$, while the remaining 9 patients will be correctly classified as carrying the mutation $(D)$

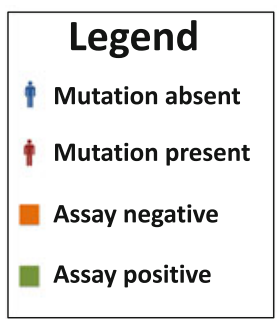

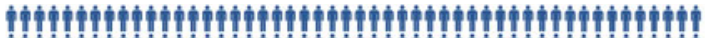

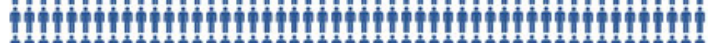

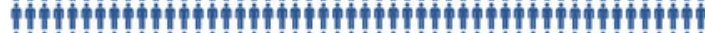

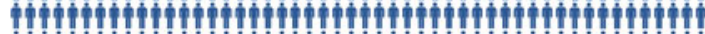

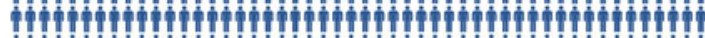

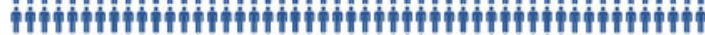

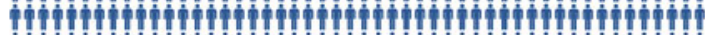

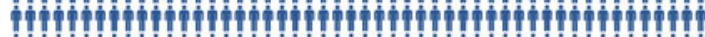

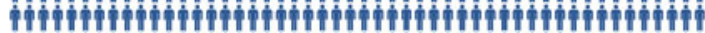

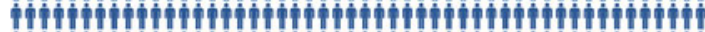

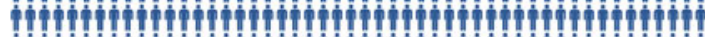

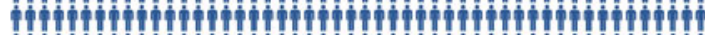

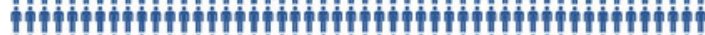

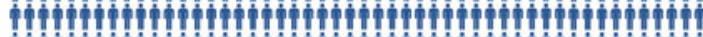

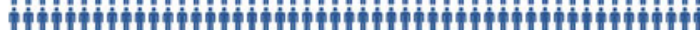

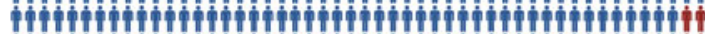

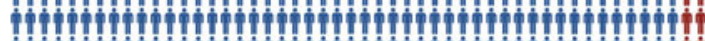

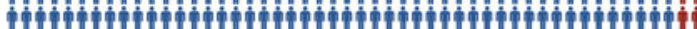

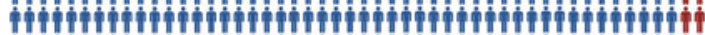

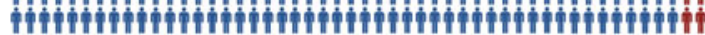

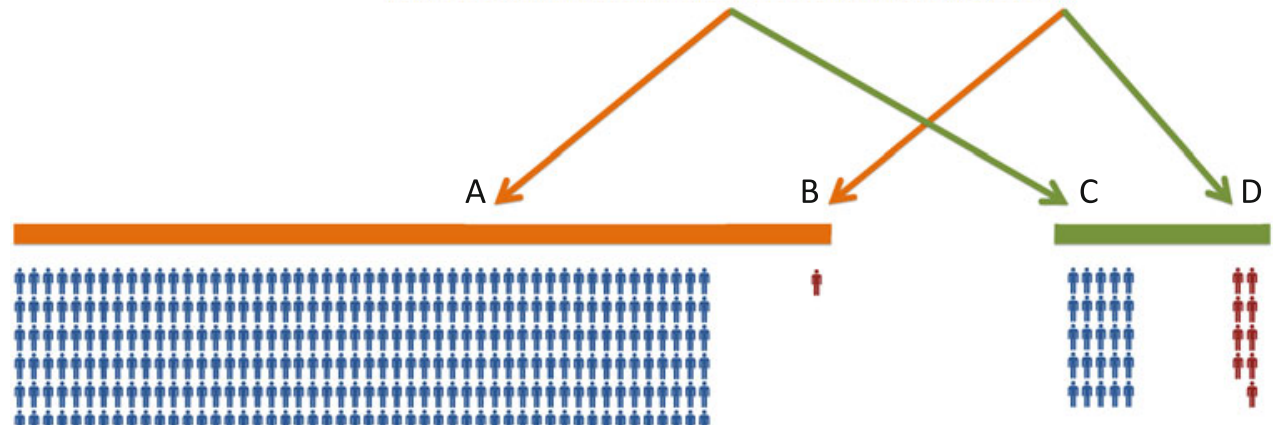

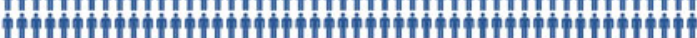

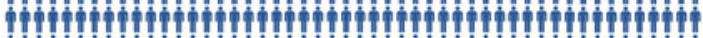

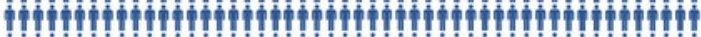
申

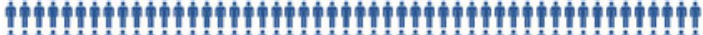

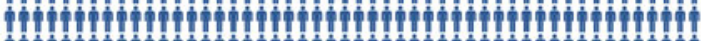

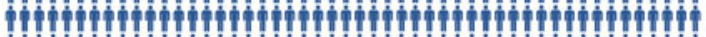

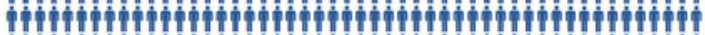

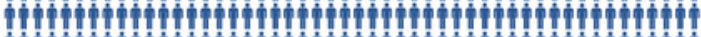

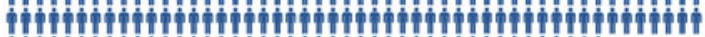

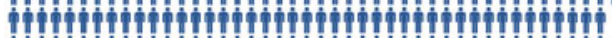

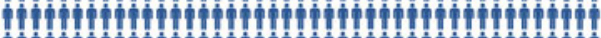

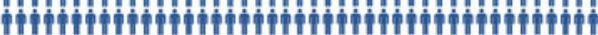

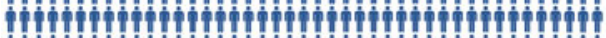

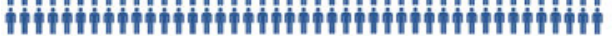


induce regression of the tumour by inhibiting the overactive BRAF protein.

The opposite of the above case is noted in $\mathrm{mCRC}$, where the presence of an activating mutation of the $B R A F$ gene has been shown to be a negative prognostic factor and also may predict a poor response to therapy with the monoclonal antibody (mAb)-based cetuximab [3-8]. Cetuximab, an anti-EGFR monoclonal antibody, operates by competing with the EGFR ligands to prevent signalling via this proinflammatory, pro-oncogenic pathway. However, as a downstream signalling molecule in the EGFR signalling pathway, an overactive BRAF protein may render a tumour resistant to $\mathrm{mAb}$ treatment targeting the EGFR.

In the context of malignant melanoma, $B R A F$ mutation testing is now standard of care, whereas in the context of mCRC it is not. However, for mCRC, it is likely that more emphasis may be placed on less frequently observed molecular aberrations, such as $B R A F$ mutation status, as evidence accumulates to describe additional causes of antiEGFR therapy resistance. Additionally, from a medical genetics perspective, $B R A F$ mutation status is widely used to differentiate between spontaneous CRC and Lynch syndrome [9]. Furthermore, the abundance of more efficient sequencing technologies may encourage the use of multi-marker panels and the increased use of off-label molecular testing to fine tune treatment decisions. This multi-marker approach to oncology brings with it many advantages, but as a corollary, presents many potential pitfalls not only for the oncologist but also for the laboratory.

To help us illustrate the context-specific predictive value of these assays we will use the sensitivity and specificity figures presented to us in the package insert of one CE-IVD marked assay in routine use for predictive testing of $B R A F$ mutations for the treatment of malignant melanoma. We are informed that these figures have a sensitivity of $99.6 \%$ and a specificity of $87.9 \%$. If an approximate $B R A F$ p.V600X mutation rate of $50 \%$ is assumed for malignant melanoma based on published reports [10-12], this would equate to a PPV of $89.2 \%$ and a NPV of $99.5 \%$. Thus, a prescribing oncologist could be reasonably certain that approximately 9 in 10 patients who are prescribed vemurafinib actually harbour the BRAF p.V600X mutation in the tested tumour sample. Depending on the side effects of the therapy, this may be considered a respectably low risk level. Similarly, the NPV for this assay in malignant melanoma $(99.5 \%)$ should allow the oncologist to be confident that any patient who is refused therapy on the basis of a negative $B R A F$ mutation test is highly unlikely to have been given a false negative result.

The opposite situation to malignant melanoma applies if $B R A F$ mutation testing using this same assay is applied to pre-testing for cetuximab or panitumumab therapy in
mCRC; that is, a positive $B R A F$ mutation result would contraindicate therapy. With an estimated prevalence of $8 \%$ in mCRC tumours $[4,7,13-15], B R A F$ mutations are approximately 7-fold less prevalent in mCRC than malignant melanoma. In this case, the clinical utility of the assay is noticeably altered; the PPV can be calculated to be $41.4 \%$ and the NPV $99.9 \%$ for the same assay used for a different condition. Thus, a clinician could only be confident that a positive result truly reflected a treatmentresistant tumour sample in approximately four out of every ten positive results. While, the NPV may give one reason to be confident that those given therapy are likely to be $B R A F$ mutation negative, one could not reliably contraindicate therapy in the case of a positive result, which would render the assay far less useful if implemented in this context. So, the implementation of BRAF mutation testing in CRC would not be a simple case of using the same assay on a different tumour type. Likely, a new or re-optimised assay dedicated for use in CRC would be required.

\section{Conclusions}

While BRAF mutation status is but a single molecular target, the implications of blindly applying an assay to a novel clinical scenario are obvious. It is important that assays are selected and optimised based on clinical rather than purely analytical properties. To this end, calculation of positive and negative predictive value in the correct clinical context may eliminate misconceptions regarding the clinical applicability of an assay. A further point that can be inferred from the above discussion is that testing of populations with low mutation prevalence rates may increase the likelihood of false positive results; thus, judicious use of molecular testing is necessary to reduce this avoidable form of error.

Acknowledgements and Disclosures The authors have no conflicts to declare that are directly relevant to the content of this article.

\section{References}

1. Gigerenzer G, Gaissmaier W, Kurz-Milcke E, et al. Helping doctors and patients make sense of health statistics. Psychol Sci Public Interest. 2008;8:53-96.

2. Da Rocha Dias S, Salmonson T, van Zwieten-Boot B, et al. The European Medicines Agency review of vemurafenib (Zelboraf ${ }^{\circledR}$ ) for the treatment of adult patients with BRAF V600 mutationpositive unresectable or metastatic melanoma: summary of the scientific assessment of the Committee for Medicinal Products for Huma. Eur J Cancer. 2013;49:1654-61.

3. Loupakis F, Ruzzo A, Cremolini C, et al. KRAS codon 61, 146 and BRAF mutations predict resistance to cetuximab plus irinotecan in KRAS codon 12 and 13 wild-type metastatic colorectal cancer. Br J Cancer. 2009;101:715-21. 
4. Richman SD, Seymour MT, Chambers P, et al. KRAS and BRAF mutations in advanced colorectal cancer are associated with poor prognosis but do not preclude benefit from oxaliplatin or irinotecan: results from the MRC FOCUS trial. J Clin Oncol. 2009;27:5931-7.

5. Borràs E, Jurado I, Hernan I, et al. Clinical pharmacogenomic testing of KRAS, BRAF and EGFR mutations by high resolution melting analysis and ultra-deep pyrosequencing. BMC Cancer. 2011;11:406.

6. Phillips B, Kalady M, Kim R. BRAF testing in advanced colorectal cancer: is it ready for prime time? Clin Adv Hematol Oncol. 2010;8:437-44.

7. De Roock W, Claes B, Bernasconi D, et al. Effects of KRAS, BRAF, NRAS, and PIK3CA mutations on the efficacy of cetuximab plus chemotherapy in chemotherapy-refractory metastatic colorectal cancer: a retrospective consortium analysis. Lancet Oncol. 2010;11:753-62.

8. Laurent-Puig P, Cayre A, Manceau G, et al. Analysis of PTEN, BRAF, and EGFR status in determining benefit from cetuximab therapy in wild-type KRAS metastatic colon cancer. J Clin Oncol. 2009;27:5924-30.
9. Bedeir A, Krasinskas AM. Molecular diagnostics of colorectal cancer. Arch Pathol Lab Med. 2011;135:578-87.

10. Yancovitz M, Litterman A, Yoon J, et al. Intra- and inter-tumor heterogeneity of $\mathrm{BRAF}(\mathrm{V} 600 \mathrm{E}))$ mutations in primary and metastatic melanoma. PLoS One. 2012;7:e29336.

11. Davies H, Bignell GR, Cox C, et al. Mutations of the BRAF gene in human cancer. Nature. 2002;417:949-54.

12. Shinozaki M. Incidence of BRAF oncogene mutation and clinical relevance for primary cutaneous melanomas. Clin Cancer Res. 2004;10:1753-7.

13. Teng H-W, Huang Y-C, Lin J-K, et al. BRAF mutation is a prognostic biomarker for colorectal liver metastasectomy. J Surg Oncol. 2012;106:123-9.

14. Tol J, Dijkstra JR, Klomp M, et al. Markers for EGFR pathway activation as predictor of outcome in metastatic colorectal cancer patients treated with or without cetuximab. Eur J Cancer. 2010;46:1997-2009.

15. Vaughn CP, Zobell SD, Furtado LV, et al. Frequency of KRAS, BRAF, and NRAS mutations in colorectal cancer. Genes Chromosomes Cancer. 2011;50:307-12. 